\title{
La elección del estudio de caso en investigación educativa
}

\section{The selection of case studies in education research}

\author{
Carmen Álvarez Álvarez \\ Profesora ayudante. Departamento de Educación. Universidad de Cantabria. \\ carmen.alvarez@unican.es
}

\author{
José Luis San Fabián Maroto \\ Catedrático de Universidad. Departamento de Ciencias de la Educación. Universidad de Oviedo. \\ jlsanfa@uniovi.es
}

\begin{abstract}
RESUMEN
Cada vez son más frecuentes los estudios de caso en la investigación educativa, pero no siempre su elección aparece suficientemente justificada. Aunque se trata de una metodología muy abierta y a priori escasamente estructurada, su adecuada utilización debe tener en cuenta algunas premisas básicas. En este artículo realizamos una aproximación conceptual y analizamos las características básicas de un estudio de caso, sus diferentes tipos, las fases de su elaboración, cómo puede asegurarse la credibilidad de los datos recogidos, así como sus principales ventajas y limitaciones. A partir de nuestra experiencia como investigadores educativos y teniendo como referencia la literatura sobre el tema, pretendemos suscitar una reflexión teórica y metodológica sobre las condiciones e implicaciones de la utilización de estudios de casos en la investigación educativa, con la finalidad de orientar su elección como método.
\end{abstract}

\begin{abstract}
Case studies are increasingly frequent in education research, but the selection criteria are not always sufficiently clear. This article presents a conceptual approach and defines the basic characteristics of a case study, the different types, stages of their development, how to ensure the credibility of the data collected, and their main advantages and disadvantages. Taking as a reference our experience as educational researchers, together with the literature on the subject, we offer a theoretical and methodological reflection on the conditions and implications of using case studies in education research, in order to guide its selection as a method.
\end{abstract}

PALABRAS CLAVE | KEYWORDS

estudios de caso | investigación educativa | metodología de investigación | investigación cualitativa | case study | education research | research methodology | qualitative research

\section{Introducción}

En los últimos años podemos constatar un notable incremento en el uso de estudios de caso en diferentes campos de las ciencias sociales, tales como la Antropología, la Psicología, la Economía, la Sociología o la Pedagogía. Cada vez proliferan más los estudios de caso, pero, en proporción a ello, aún son escasos los trabajos que se ocupan de sistematizar las características, propiedades y exigencias propias de este método de investigación. De hecho, apenas existen libros dirigidos a teorizar sobre el mismo, siendo los trabajos más citados y más exhaustivos los de Yin (1989), Stake (2005) y Grandon Gill's (2011) en el panorama internacional, sentando las bases teóricas y prácticas del método. También han realizado aportaciones significativas a este método, a nivel de metodología, otros trabajos producidos en el ámbito internacional como los de Hammersley (1986), Ragin y Becker (1992), Hamel y otros (1993), Merriam (1998) y Bassey (2000).

En el contexto español cabría destacar los trabajos de Martínez Bonafé (1990), López-Barajas Zayas (1996), Angulo y Vázquez (2003) o Coller (2005). En todos ellos es posible adentrarse en los 
fundamentos teóricos y metodológicos a seguir en los estudios de casos y encontrar algunos ejemplos. Además, en España, en algunos manuales sobre investigación educativa pueden encontrarse alusiones a este marco metodológico como en Cohen y Manion (1990), Cajide (1992), Arnal, Del Rincón y Latorre (1994), Pérez Serrano (1994), Rodríguez Gómez y otros (1996), Ander Egg (2000), Taylor y Bodgan (2000), Bell (2002), Cardona Moltó (2002), así como en algunos artículos, tales como los de Walker (1983), Bonache (1999), Rodríguez Rojo (2000), Chavarría Navarro, Hampshire y Martínez (2004) y Cebreiro López y Fernández Morante (2004).También es posible encontrar referencias metodológicas en estudios temáticos (Marcelo, 1991; Heras Montoya, 1997; Contreras y otros 2000), si bien la mayoría de los investigadores que realizan estudios de caso no se detienen a establecer pautas metodológicas.

Toda la investigación y difusión precedente sobre estudios de caso indican que no estamos ante un método nuevo de investigación. Sin embargo, el mismo no ha perdido interés, ni vigencia, todo lo contrario: en ciencias sociales cada vez se hacen más estudios de caso, pero: ¿por qué se elige el estudio de caso como método para investigar?

A partir de la literatura sobre la metodología de estudio de caso y de nuestra propia participación en varios trabajos en los que se emplea este método de investigación tratamos de responder las siguientes preguntas en este artículo:

- ¿Qué caracteriza un estudio de caso en relación a otros métodos?

- ¿Por qué elegir el estudio de caso como marco metodológico de una investigación?

- ¿Qué modalidad de estudio de caso es la más adecuada en una investigación concreta?

- ¿Cómo proceder para elaborar un estudio de caso?

- ¿Cómo asegurar el rigor de los datos, procesos y resultados?

\section{1. ¿Qué caracteriza a un estudio de caso?}

El estudio de caso destaca entre los diseños de tipo cualitativo, junto con los de investigación-acción y los estudios etnográficos, con los que se confunde con frecuencia (Expósito y otros 2004). En algunas disciplinas de administración es el método cualitativo de investigación más utilizado (Yacuzzi 2005). Dado el incremento que ha experimentado la realización de estudios de caso en investigación parece conveniente plantear su delimitación, pues no siempre se discrimina bien lo que es.

Aunque compartan algunos rasgos en común, aquí nos referimos al estudio de caso como metodología de investigación, no como instrumento de análisis o de diagnóstico previo a la intervención jurídica, clínica o educativa (Crespo 2000) ni como herramienta didáctica al servicio del aprendizaje activo (Martínez y Musitu 1995). Se trata de una metodología vinculada a la sociología clásica a través de autores como Spencer, Weber o Merton y que tiene su punto álgido en la Escuela de Chicago.

El estudio de caso -o estudio/s de casos, dependiendo de los autores- es un concepto que abarca numerosas concepciones sobre la investigación. De hecho, es un término que sirve de "paraguas" para toda una amplia familia de métodos de investigación cuya característica básica es la indagación en torno a un ejemplo. Stake (2005: 12) plantea esta cuestión cuando asevera que "existen muchísimas formas de hacer estudios de casos".

Desde el diseño hasta la presentación de sus resultados, el método de casos está estrechamente vinculado con la teoría. Según Yacuzzi, los casos de investigación son especialmente valiosos, porque permiten el estudio de la causalidad y la plasman en una teoría. "Todo buen diseño incorpora una teoría, que sirve como plano general de la investigación, de la búsqueda de datos y de su interpretación. A medida que el caso se desarrolla, emerge una teoría más madura, que se va cristalizando (aunque no necesariamente con perfección) hasta que el caso concluye" (Yacuzzi 2005: 9). Para algunos autores su potencialidad radica en su capacidad para generar premisas hipotéticas y orientar la toma de decisiones. Así, para 
Arnal, Del Rincón y Latorre el estudio de casos "debe considerarse como una estrategia encaminada a la toma de decisiones. Su verdadero poder radica en su capacidad para generar hipótesis y descubrimientos, en centrar su interés en un individuo, evento o institución, y en su flexibilidad y aplicabilidad a situaciones naturales" (Arnal, Del Rincón y Latorre 1994: 206).

Según Stake (2005: 11), la nota distintiva del estudio de casos está en la comprensión de la realidad objeto de estudio: "El estudio de casos es el estudio de la particularidad y de la complejidad de un caso singular, para llegar a comprender su actividad en circunstancias importantes". Desde una perspectiva interpretativa, Pérez Serrano (1994: 81) afirma que "su objetivo básico es comprender el significado de una experiencia". El conocimiento de lo particular, de lo idiosincrásico, sin olvidar su contexto, parece que está presente en la intencionalidad de la investigación basada en estudios de caso. Autores como Yin (1989) enfatizan la contextualización del objeto de investigación, al entender que un estudio de caso es una investigación empírica dirigida a investigar un fenómeno contemporáneo dentro de su contexto real por la imposibilidad de separar a las variables de estudio de su contexto. Walker (1983: 45) viene a reforzar esta idea dinámica cuando apunta que un estudio de casos "es el examen de un ejemplo en acción". La metodología del estudio de caso aplicada a la investigación social remite inevitablemente a la etnografía.

Suele hacerse hincapié en el proceso descriptivo y holístico que caracteriza esta metodología, dirigiéndose a la comprensión holística de un sistema cultural en acción (Feagin, Orum y Sjoberg, 1991). Pérez Serrano define al estudio de casos como "una descripción intensiva, holística y un análisis de una entidad singular, un fenómeno o unidad social. Los estudios de casos son particularistas, descriptivos y heurísticos y se basan en el razonamiento inductivo al manejar múltiples fuentes de datos" (Pérez Serrano 1994: 85). La potencia y justificación investigadora de un estudio de caso se basa en el supuesto de que lo global se refleja en lo local (Hamel y otros 1993), es decir, en la naturaleza holográfica de la realidad, describiendo cualquier proceso de una unidad de vida en sus diversas interrelaciones con su escenario cultural.

Es a una visión global del fenómeno estudiado a lo que aspira un estudio de caso. No es una técnica particular para conseguir datos sino una forma de organizarlos (Goode y Hatt, 1976). Cebreiro López y Fernández Morante (2004: 665) consideran que su aportación al estudio de los fenómenos educativos se fundamenta en tres rasgos:

1. Énfasis en las observaciones a largo plazo, basadas más en informes descriptivos que en categorías pre-establecidas.

2. Interés por describir la conducta observada, dentro del marco de los hechos circundantes.

3. Una preocupación por la perspectiva de los participantes acerca de los hechos, es decir, cómo construyen su realidad social.

Hablar de estudio de casos es hablar de un método que abarca una diversidad de fuentes y técnicas de recogida de información. Cebreiro López y Fernández Morante enfatizan esta cuestión cuando afirman que:

"Mediante este método, se recogen de forma descriptiva distintos tipos de informaciones cualitativas, que no aparecen reflejadas en números si no en palabras. Lo esencial en esta metodología es poner de relieve incidentes clave, en términos descriptivos, mediante el uso de entrevistas, notas de campo, observaciones, grabaciones de vídeo, documentos" (Cebreiro López y Fernández Morante 2004: 666).

Teniendo en cuenta las aportaciones de diferentes autores y a partir también de nuestra experiencia investigadora, podemos destacar un conjunto de características básicas de los estudios de casos, que, en su conjunto, les diferencia de otros métodos de investigación:

1. Realizan una descripción contextualizada del objeto de estudio. El principal valor de un estudio 
de caso consiste en desvelar las relaciones entre una situación particular y su contexto.

2. Son estudios holísticos. El investigador ha de tratar de observar la realidad con una visión profunda y, asimismo, ha de tratar de ofrecer una visión total del fenómeno objeto de estudio, reflejando la complejidad del mismo.

3. Reflejan la peculiaridad y la particularidad de cada realidad/situación a través de una descripción densa y fiel del fenómeno investigado.

4. Son heurísticos. Los estudios de caso tratan de iluminar la comprensión del lector sobre el fenómeno social objeto de estudio.

5. Su enfoque no es hipotético. Se observa, se sacan conclusiones y se informa de ellas.

6. Se centran en las relaciones y las interacciones y, por tanto, exigen la participación del investigador en el devenir del caso.

7. Estudian fenómenos contemporáneos analizando un aspecto de interés de los mismos, exigiendo al investigador una permanencia en el campo prolongada.

8. Se dan procesos de negociación entre el investigador y los participantes de forma permanente.

9. Los estudios de caso incorporan múltiples fuentes de datos y el análisis de los mismos se ha de realizar de modo global e interrelacionado.

10. El razonamiento es inductivo. Las premisas y la expansión de los resultados a otros casos surgen fundamentalmente del trabajo de campo, lo que exige una descripción minuciosa del proceso investigador seguido.

Estas características, no siempre se cumplen en todos los estudios de caso que se realizan actualmente, lo que nos lleva a plantearnos si no se estará abusando del término "estudio de caso" usándolo para designar todo tipo de investigaciones en las que la muestra a examinar es una $(\mathrm{N}=1)$.

\section{2. ¿Por qué elegir el estudio de caso en una investigación?}

Cebreiro López y Fernández Morante afirman que es conveniente desarrollar un estudio de caso "cuando el objeto que se quiere indagar está difuso, es complejo, escurridizo o controvertido. Es decir, para analizar aquellos problemas o situaciones que presentan múltiples variables y que están estrechamente vinculados al contexto en el que se desarrollan" (Cebreiro López y Fernández Morante 2004: 667). Según Yacuzzi (2005), los casos son particularmente válidos cuando se presentan preguntas del tipo "cómo" o "por qué", cuando el investigador tiene poco control sobre los acontecimientos y cuando el problema a estudiar es incipiente.

Por otro lado, la elección de un estudio de casos puede apoyarse en tres razones (Rodríguez y otros 1996 : 95):

1. Su carácter crítico, es decir, en la medida en que el caso permite confirmar, cambiar, modificar o ampliar el conocimiento sobre el objeto de estudio.

2. Su carácter extremo o unicidad, pues parte de una situación que tiene un carácter específico y peculiar. Como señala Stake, "estudiamos un caso cuando tiene un interés muy especial en sí mismo" (Stake 2005: 11).

3. Finalmente, el carácter revelador del caso permite observar y analizar un fenómeno o hecho particular relativamente desconocido en la investigación educativa y sobre el cual pueden realizarse aportaciones de enorme relevancia.

Rodríguez Gómez y otros (1996: 95) sugieren otros criterios complementarios de utilidad, aunque secundarios, a la hora de seleccionar casos, tales como:

- Facilidad para acceder al mismo y/ o permanecer en el campo todo el tiempo que sea necesario.

- Existencia de una alta probabilidad de que se dé una mezcla de procesos, programas, personas, interacciones y/ o estructuras relacionadas con las cuestiones de investigación. 
- Posibilidad de establecer una buena relación con los informantes.

- Poder asegurar la calidad y credibilidad del estudio.

El éxito de los estudios de caso guarda una estrecha relación con el cumplimiento de una serie de requisitos o condiciones como el interés e implicación de los sujetos investigados en el estudio a realizar, las buenas relaciones entre investigadores y sujetos investigados, la probabilidad de innovar o transformar la situación de partida con la investigación o la ventaja indudable de contar con apoyos (asesores) en los procesos educativos de cambio.

La realización de estudios de caso en la investigación educativa plantea ventajas y también algunas limitaciones con las que debemos contar y paliar en la medida de lo posible. Un estudio de caso abre enormes posibilidades a la investigación, tales como:

1. Permite descubrir hechos o procesos que si se utilizasen otros métodos probablemente se pasarían por alto, arrojando luz sobre cuestiones sutiles (Walker 1983: 42-59; Arnal y otros 1994: 209; Stake 1995: 48-49).

2. Ayuda a desvelar significados profundos y desconocidos, así como orientar la toma de decisiones en relación a problemáticas educativas (Bell 2002: 22-23; Heras Montoya 1997: 121-124; Pérez Serrano 1994: 99-102).

3. Es valioso para informar de realidades educativas complejas, invisibilizadas por la cotidianeidad, para entender procesos internos y descubrir dilemas y contradicciones, ayudando a reflexionar sobre las prácticas.

4. Aporta concreción, intensidad y detalle respecto al tema de estudio, al explorar lo más profundo de una experiencia.

5. Es posible emplear una diversa gama de técnicas en la recogida y análisis de datos, tanto cuantitativos como cualitativos (Pérez Serrano 1994: 99-102; Rodríguez Gómez y otros 1996: 91-92; Cebreiro López y Fernández Morante 2004: 667).

6. Permite, y requiere, la triangulación de la información recogida para evitar el sesgo del investigador (Arnal y otros 1994: 209; Pérez Serrano 1994: 99-102; Cebreiro López y Fernández Morante 2004: 667; Stake 1995: 48-49). .

7. Se considera un método muy adecuado para investigadores individuales y a pequeña escala.

Por otra parte, Pérez Serrano (1994: 116-118) plantea que la elaboración de estudio de casos conlleva valiosas potencialidades formativas para la persona que lo realiza, tales como:

1. Adquisición de experiencia en el diagnóstico de problemas concretos.

2. Una comprensión más completa y realista de la realidad.

3. Desarrollo de la capacidad de análisis y de síntesis.

4. Capacidad para pensar de forma lógica y rápida.

5. Integración -interrelación- de conocimientos y vivencias.

6. Motivación.

Pese a sus bondades, los estudios de caso también han sido objeto de críticas, especialmente desde la investigación cuantitativa, que observa en ellos un conjunto de limitaciones:

a) La imposibilidad de generalizar los resultados obtenidos en la investigación. Claramente el objetivo de esta metodología es otro, el de profundizar en una o varias unidades de observación. Walker, al respecto, opina que generalizar no es un problema para el investigador que realiza un estudio de casos. "Es el lector quien tiene que preguntarse: ¿Qué existe en ese estudio que yo pueda aplicar a mi propia situación y qué es lo que claramente no se aplica?" (Walker 1983: 47). No obstante, también existen estudios por acumulación (multi-casos) que se realizan a partir del análisis de varios estudios de casos. De cualquier forma, teniendo en cuenta el paradigma cualitativo en el que se enmarca esta metodología, no parece oportuno hablar de "generalización" sino más bien de "transferencia". Frente a la generalización estadística de la investigación cuantitativa, el estudio de caso persigue la "generalización 
analítica" (Yin 1989), donde la teoría generada es usada como patrón con el cual comparar los resultados de otros estudios. El estudio de caso permite la transferencia "hacia la teoría" y no hacia otros casos (Yacuzzi 2005), siendo lo realmente importante la generación de ideas aprovechables en diferentes escenarios. Martínez Carazo señala que "el método de estudio de caso es una estrategia metodológica de investigación científica, útil en la generación de resultados que posibilitan el fortalecimiento, crecimiento y desarrollo de teorías existentes o el surgimiento de nuevos paradigmas científicos" (Martínez Carazo 2006: 189-190).

b) La subjetividad del investigador presente a lo largo del estudio. Esta cuestión no siempre es vista como un problema por parte de los investigadores: "Se sabe que la pretensión de los investigadores cualitativos es realizar una investigación subjetiva. No se considera que la subjetividad sea un fallo que hay que eliminar, sino un elemento esencial de comprensión" (Stake 1995. 48). Frecuentemente se argumenta que se ejerce una "subjetividad controlada" por parte del investigador, y que la puesta en relación de ésta y la de los implicados produce una situación denominada de "intersubjetividad" que aporta una gran riqueza a los estudios. La credibilidad de los datos cualitativos depende en gran medida de la formación, sensibilidad, habilidades metodológicas y capacidad ética del investigador (Patton 1987), también, debemos añadir, del carácter explícito de sus motivaciones y procedimientos. Para evitar que la propia subjetividad sea perjudicial para la investigación, deben mostrarse permanentemente las preocupaciones respecto a la investigación y describirse minuciosamente las intervenciones desarrolladas con el objeto de favorecer la replicabilidad del estudio.

c) La posibilidad de alterar el modo de vida de los sujetos estudiados al permanecer en el campo durante un tiempo. El acceso negociado, el contacto directo y la permanencia duradera en el campo de estudio por parte del investigador son requisitos de esta metodología. En un primer momento es posible que el investigador pueda condicionar el modo de vida de los sujetos sobre los que se indaga, pero su objetivo es llegar a ser uno más en el grupo social, de forma que los sujetos se acostumbren a su presencia.

\section{3. ¿Qué modalidad de estudio de caso es la más adecuada?}

El estudio de caso es todo lo contrario a una metodología uniforme, se adapta a cada realidad y adquiere modalidades específicas en función de su contexto y finalidad, de aquí la importancia de encontrar la modalidad adecuada.

Ragin y Becker (1992) distinguen diferentes formas de enfocar un caso según se consideren como unidades empíricas o construcciones teóricas, de forma que pueden ser considerados: realidades encontradas o descubiertas, objetos, hechos o convenciones. En función de la disponibilidad de una teoría previa más elaborada por parte del investigador, los casos pueden ser exploratorios, explicativos o descriptivos. A su vez, los diseños pueden ser de un caso simple o de múltiples casos y, por otra parte, holísticos o encapsulados, según se utilice una o varias unidades de análisis (Yin 1989).

$\mathrm{Si}$ atendemos a la finalidad del estudio de caso, a las técnicas de recogida de información y al informe, podemos concluir que no existe un único modo de hacer estudios de caso. Stake (2005) plantea que hay tres tipos de estudios de caso atendiendo a la finalidad última del mismo:

- Estudio de caso intrínseco: casos con especificidades propias, que tienen un valor en sí mismos y pretenden alcanzar una mejor comprensión del caso concreto a estudiar. En este supuesto no se elige al caso porque sea representativo de otros casos, o porque ilustre un determinado problema o rasgo, sino porque el caso en sí es de interés. Yin (1989) se refiere a él como diseño de caso único.

- Estudio de caso instrumental: al servicio de la construcción de una teoría. Son casos que pretenden generalizar a partir de un conjunto de situaciones específicas. El caso se examina para profundizar en un tema o afinar una teoría, de tal modo que el caso juega un papel secundario, de 
apoyo, para llegar a la formulación de afirmaciones sobre el objeto de estudio. Es el diseño de casos múltiples y se emplea cuando se dispone de varios casos para replicar.

- Estudio de caso colectivo: se realiza cuando el interés de la investigación se centra en un fenómeno, población o condición general seleccionando para ello varios casos que se han de estudiar intensivamente.

Heras Montoya (1997), atendiendo al objeto del estudio, su perspectiva y las técnicas de recogida de información a emplear, ofrece una clasificación diferente:

1. Estudio organizativo histórico: se centra en la evolución en el tiempo de una organización con una perspectiva diacrónica empleando técnicas como la entrevista y el análisis de documentos.

2. Estudio de historias de vida: se examina una persona para emplear los datos que ofrezca como vehículo para entender aspectos básicos de la conducta humana o de alguna institución actual. La perspectiva es diacrónica y la técnica más importante es la entrevista.

3. Estudio observacional: el foco de estudio es una organización o un aspecto determinado de la misma tal y cómo es en su estado actual. Se realiza con una perspectiva sincrónica y la técnica más relevante es la observación participante.

Pérez Serrano (1994) plantea estos tipos de estudios de casos, atendiendo fundamentalmente al informe de investigación:

1. Estudio de casos descriptivo: se presenta un informe detallado del fenómeno objeto de estudio sin fundamentación teórica previa, con el objeto de no guiarse por hipótesis preestablecidas y aportar información básica sobre áreas educativas no investigadas aún.

2. Estudio de casos interpretativo: contiene descripciones ricas y densas, sin embargo, difiere del anterior en que los datos se utilizan para desarrollar categorías conceptuales o para ilustrar, defender o desafiar presupuestos teóricos defendidos antes de recoger los datos.

3. Estudio de casos evaluativo: implican descripción y explicación para llegar a emitir juicios sobre la realidad objeto de estudio.

Un mismo estudio de caso puede recoger a su vez varias modalidades, en función del objeto y desarrollo de la investigación. No existe una estructura metodológica estándar para ser aplicada y, aunque haya una base común, la propuesta metodológica resultante debe ser en cierto sentido única.

\section{4. ¿Cómo proceder para elaborar un estudio de caso?}

Según Yin (1989), un diseño de investigación se compone de cinco componentes: las preguntas del estudio, las proposiciones, si existieran, su unidad de análisis (pueden ser varias), la lógica que vincula los datos con las proposiciones y los criterios para interpretar los hallazgos.

La investigación mediante estudios de casos sigue unas fases generales ampliamente aceptadas. Tomando la clasificación de Pérez Serrano (1994) y Martínez Bonafé (1990) podemos distinguir entre:

1. Fase preactiva. En ella se tienen en cuenta los fundamentos epistemológicos que enmarcan el problema o caso, los objetivos pretendidos, la información de que se dispone, los criterios de selección de los casos, las influencias del contexto donde se han desarrollado los estudios, los recursos y las técnicas que se han de necesitar y una temporalización aproximada. La pregunta de investigación sirve para definir la unidad o unidades de análisis a considerar. De esta forma, se establece una relación entre constructos teóricos y unidades empíricas, categorías generales y específicas (Ragin y Becker 1992), estableciendo una "cadena de evidencias" (Yin 1989).

Una de las tareas más difíciles es esclarecer empíricamente el tamaño y los límites de un determinado 
caso. También lo es establecer el número de eventos o situaciones que permitan atribuir a una muestra de registros la representatividad del conjunto (Carbaugh 2007). Desde la investigación cualitativa, los investigadores plantean que en esta fase tienen que enfrentarse a problemas prácticos tales como:

- Desconocer el ámbito de estudio concreto en el que se desarrollará la investigación (el contexto institucional y social del ámbito educativo seleccionado, por ejemplo).

- Plantearse a priori todo el proceso de investigación (cronología, estrategias de recogida de datos, etc.).

2. Fase interactiva. Corresponde al trabajo de campo y a los procedimientos y desarrollo del estudio, utilizando diferentes técnicas cualitativas: toma de contacto y negociación que sirven para delimitar las perspectivas iniciales del investigador, las entrevistas, la observación y las evidencias documentales. En esta fase es fundamental el procedimiento de la triangulación para que pueda ser contrastada la información desde fuentes diferentes.

En la fase interactiva la principal preocupación suele ser recoger, reducir y relacionar la información recogida a través de diferentes técnicas: observación participante, entrevista, foros de debate y análisis documental. En ocasiones esta fase se vuelve problemática porque:

- El investigador puede tender a implicarse en exceso en las cuestiones que investiga o bien tener dificultades para empatizar con el grupo.

- Pueden surgir problemas de tiempo, como disponer de poco tiempo para realizar el trabajo de campo, tener que adaptarse a un horario poco conveniente, etc.

- El investigador juega un papel "temporal" en el contexto a investigar (mientras dure la investigación).

- El volumen de datos recogidos suele ser muy amplio y difícil de reducir.

- No es fácil lograr el equilibrio entre la visión microscópica y la macroscópica.

Un método como éste, que pone al investigador en contacto directo y prolongado con la realidad estudiada, requiere herramientas heurísticas para detectar los cambios y movimientos que sufre esa realidad. El uso de tipologías será fundamental para ordenar y relacionar los registros que permitan observar los cambios e identificar su dirección a través del tiempo.

- El modo teórico: ordena la realidad a partir de esquemas teóricos explicativos existentes.

- El modo descriptivo: se apoya en el registro minucioso de la realidad a través de algún artefacto académico.

- El modo de interpretación: selecciona y organiza los datos en un conjunto de significaciones culturales.

- El modo comparativo: se pregunta por la existencia de prácticas similares en otros contextos.

- El modo crítico: indaga en los desequilibrios y desigualdades vinculadas a las estructuras de poder existentes.

3. Fase postactiva. Se refiere a la elaboración del informe del estudio final en que se detallan las reflexiones críticas sobre el problema o caso estudiado. Algunos problemas prácticos propios de esta fase son:

- Dificultades respecto a la confidencialidad de los datos, pues debe salvaguardarse la identidad de aquellos sujetos que se investigan, salvo que en el estudio se acuerde que es importante desvelar algunos datos identificativos.

- Escasez de tiempo por tener que efectuar una redacción y entrega de informes ágil.

- Problemas a la hora de difundir los resultados en la comunidad de profesionales y en la comunidad científica, también en los medios de comunicación social. 


\section{5. ¿Cómo asegurar el rigor de los datos, procesos y resultados?}

La credibilidad de la investigación cualitativa mediante estudios de caso ha sido una preocupación insistente en el contexto de la investigación educativa general, tildándose a aquella con frecuencia de imprecisa, subjetiva o distorsionada. Para juzgar la credibilidad de un estudio de caso debemos hacernos las siguientes preguntas:

1. ¿Cómo se ha recogido la información? ¿qué fuentes de información se emplean? ¿cómo ha sido procesada y transcrita e incorporada a la perspectiva teórica del investigador la información recogida?

2. ¿Cómo puede ser verificada y confirmada la información? ¿cuál es fidelidad a las perspectivas de los informantes? ¿se han respetado los derechos de los participantes?

3. ¿Cómo se ha interpretado la información? ¿cómo se ha llegado a las conclusiones y se han emitido los juicios?

Para garantizar la credibilidad de los datos existen varias estrategias a tener en cuenta. Destacamos entre ellas la contextualización, la saturación, la negociación con los implicados y la triangulación.

\section{Contextualización}

Los estudios de caso de orientación etnográfica buscan analizar y comprender cómo las acciones humanas se relacionan con el contexto social en el que ocurren, por lo que la unidad de análisis debe examinarse en su entorno social y cultural.

"Suministrar contexto es ir mostrando las reglas que siguen los agentes de un modo de vida particular, proponiendo progresivamente nuevas ampliaciones ante sucesivas excepciones. Suministrar contexto también es dar la oportunidad al lector de la etnografía de ponerse en el lugar de aquéllos que viven una forma extraña de experiencia, ofreciéndole, de una manera ordenada, la mayor cantidad posible de claves significativas sobre su realidad concreta" (Velasco y Díaz de Rada, 2006: 236-237).

\section{Saturación}

La saturación guarda relación con la justificación de una afirmación apoyándose en múltiples pruebas. Si sobre un tópico se desea indagar especialmente puede que sea preciso observarlo, preguntar a los informantes más adecuados sobre él, analizar los documentos que se han generado sobre el mismo, incluso puede que sea necesario repetir estas estrategias, con el objeto de agotar las estrategias de búsqueda sobre el mismo, tratando de ver si los resultados obtenidos se mantienen en el tiempo.

\section{Negociación con los implicados}

La negociación con las personas implicadas guarda relación con el encuentro entre los fines, los métodos y los resultados del investigador y la opinión de los implicados, con el objeto de saber si hay acuerdo entre ellos, especialmente en lo que se refiere a la elaboración final del informe. Para tal cuestión pueden organizarse foros, reuniones, etc. en los que se discutan cuestiones vinculadas a las diferentes fases del estudio o en las que se revisen escritos previos a la difusión del informe. González Riaño afirma que, "cuando las personas que intervienen en el control de la información la aceptan como justa, relevante y precisa, de algún modo están asegurando la validez o credibilidad de la misma" (González Riaño 1994: 


\section{Triangulación}

La triangulación es la estrategia de validación de los datos más empleada y más conocida por los investigadores sociales. Básicamente puede entenderse como la puesta en relación de las perspectivas de los diferentes agentes implicados en la investigación, incluido el investigador. Arias Valencia plantea que: "la principal meta de la triangulación es controlar el sesgo personal de los investigadores y cubrir las deficiencias intrínsecas de un investigador singular o una teoría única, o un mismo método de estudio y así incrementar la validez de los resultados" (Arias Valencia 2000: 8) Podemos diferenciar tres tipos de triangulación:

- de métodos: cotejando la información obtenida a través de una técnica (la observación, por ejemplo) con otras (la entrevista, la revisión documental, etc.);

- de sujetos: contrastando los puntos de vista de los miembros de la comunidad estudiada;

- de espacios y tiempos: aplicando las técnicas de recogida de información (observación, entrevista y análisis documental) en diferentes espacios y tiempos, para comprobar si los resultados obtenidos son consistentes.

Finalmente, puede que lo más difícil sea escribir el caso. Un estudio de caso busca crear un relato global que se construye utilizando relatos parciales. Por eso, su base es la investigación narrativa, donde los relatos son los mimbres que permiten construir el cesto. El investigador debe buscar el orden en que debe entrelazar esos mimbres y, sobre todo, cuáles de ellos actúan como guía y soporte del resto (Moen 2006). La dificultad reside en cómo organizar los distintos significados y experiencias documentadas en el trabajo de campo, cómo convertir los relatos narrativos parciales en un relato único. La recogida y análisis de información lleva a descomponer la realidad en varios elementos o relatos, que suponen una visión global e integrada en sí mismos pero que compiten con otros para formar una visión general de la realidad. Por muy exhaustiva que sea su mirada, el investigador solo accede a determinadas parcelas de la realidad, que como tales configurarán su relato final con pretensiones de realidad total.

Investigar con estudios de caso requiere habilidades prácticas que se van adquiriendo mediante la experiencia en el campo y la práctica narrativa. Es esta experiencia la que nos dice que junto con la disciplina, la estrategia y el rigor metodológico necesario en toda investigación el estudio de caso pone a prueba la visión, la intuición y el saber hacer del oficio investigador. Se trata de un método apropiado para su desarrollo en una etapa de madurez investigadora, al exigir un equilibrio complejo entre el dominio de marcos teóricos, la descripción densa y el análisis contextual, amalgamados por el arte de saber narrar.

\section{Bibliografía}

Ander Egg, E.

2000 Métodos y técnicas de investigación social. Buenos Aires, Lumen-Humanitas.

Angulo, J. F. (y R. Vázquez)

2003 Introducción a los estudios de casos. Málaga, Aljibe.

Arias Valencia, M. M.

2000 "La triangulación metodológica: sus principios, alcances y limitaciones", Investigación y 
Educación en Enfermería, 1.

Arnak, J . (D. Del Rincón y A. Latorre)

1994 Investigación educativa. Fundamentos y metodología. Barcelona, Labor.

Bassey, $\mathrm{M}$.

2000 Case study research in educational settings. Buckinghan, Open University Press.

Bell, J .

2002 Cómo hacer tu primer trabajo de investigación. Guía para investigadores en educación y ciencias sociales. Barcelona, Gedisa.

Bonache, J .

1999 "El estudio de casos como estrategia de construcción teórica: características, críticas y defensas", Cuadernos de Economía y Dirección de la Empresa, 3: 123-140.

Cardona Moltó, M. C.

2002 Introducción a los métodos de investigación educativa. Madrid, EOS.

Cajide, $\mathrm{J}$.

1992 "La investigación cualitativa: tradiciones y perspectivas contemporáneas", Bordón, 44, 4: 357-373.

Carbaugh, D.

2007 "Cultural discourse analysis: Communication practices and intercultural encounters", J ournal of Intercultural Communication Research, 36 (3): 167-182.

Cebreiro López, B. (y M. C. Fernández Morante)

2004 "Estudio de casos", en F. Salvador Mata, J . L. Rodríguez Diéguez y A. Bolívar Botia, Diccionario enciclopédico de didáctica. Málaga, Aljibe.

Chavarría Navarro, X. (S. Hampshire y F. Martínez)

2004 "Una aproximación a los estudios de caso desde la práctica", Revista de Investigación Educativa, 22: 443-458.

Cohen, L. (y L. Manion)

1990 Métodos de investigación educativa. Madrid, La Muralla.

Coller, X.

2005 Estudio de casos. Madrid, Centro de Investigaciones Sociológicas.

Contreras, J ., (A. García Martínez y A. Rivas)

2000 Tristes institutos. Una exploración antropológica de un instituto de enseñanza secundaria y su entorno. Gijón, Fundación de Cultura de Gijón.

Crespo, R. F.

2000 "The Epistemological Status of Managerial Knowledge and the Case Method", Second ISBEE

World Congress "The Ethical Challenges of Globalization", Proceedings Latin America: 210-218.

Expósito, J . (E. Olmedo y A. Fernández Cano)

2004 "Patrones metodológicos en la investigación española sobre evaluación de programas educativos", Revista Electrónica de Investigación y Evaluación Educativa, 10, 2.

Feagin, J . (A. Orum y G. Sjoberg) (eds.)

1991 A case for case study. Chapel Hill, University of North Carolina Press. 
González Riaño, X. A.

1994 Interferencia lingüística y escuela asturiana. Oviedo, Academia de la Llingua Asturiana.

Goode, W. (y P. Hatt)

1976 Métodos de investigación social. México, Trillas.

Grandon Gill's, T.

2011 Book Informing with the Case Method. London, Informing Science Press.

Hamel, J . (S. Dufour y D. Fortin)

1993 Case study methods. California, Sage Publications.

Hammersley, M.

1986 Case studies in classroom research: a reader. Milton Keynes, Open University Press.

Heras Montoya, L.

1997 Comprender el espacio educativo. Investigación etnográfica sobre un centro escolar. Málaga, Aljibe.

J orrín Abellan, I. M. (y otros)

2007 "Lo que el ojo no ve: un estudio de caso basado en procesos de indagación co(wiki)laborativos",

Revista Latinoamericana de Educación a Distancia RIED, 10, 2: 75-96.

López-Barajas Zayas, E.

1996 El estudio de casos. Fundamentos y metodología. Madrid, UNED.

Marcelo, C. (ed.)

1991 El estudio de caso en la formación del profesorado y la investigación didáctica. Sevilla, Servicio de publicaciones de la Universidad de Sevilla.

Martíneza Bonafé, J .

1990 "El estudio de casos en la investigación cualitativa", en J . B. Martínez Rodríguez, Hacia un enfoque interpretativo de la enseñanza (57-68). Granada, Servicio de Publicaciones de la Universidad de Granada.

Martínez Carazo, P. C.

2006 "El método de estudio de caso. Estrategia metodológica de la investigación científica",

Pensamiento y Gestión, 20: 165-193.

Martínez, A. (y G. Musitu) (coords.)

1995 El estudio de casos para profesionales de la acción social. Madrid, Narcea.

Merriam, S. B.

1998 Qualitative research and case study applications in education. San Francisco, J ossey-Bass.

Moen, T.

2006 "Reflections on the narrative research approach", International J ournal of Qualitative

Methodology, 5(4).

Patton, M. Q.

1987 How to use qualitative methods in evaluation. Beverly Hills, Sage.

Pérez Serrano, G.

1994 Investigación cualitativa. Retos, interrogantes y métodos. España, La Muralla. 
Ragin, C. (y H. Becker)

1992 What is a case? Exploring the foundations of social inquiry. New York, Cambridge University Press.

Rodríguez Gómez, G. (J . Gil Flores y E. García Jiménez)

1996 Metodología de la investigación cualitativa. Málaga, Aljibe.

Rodríguez Rojo, M.

2000 "Credibilidad de la investigación cualitativa o reflexiones sobre qué y cómo observar en un estudio de casos", Revista Interuniversitaria de Formación del Profesorado, 39: 119-130.

Stake, R. E.

2005 Investigación con estudio de casos. Madrid, Morata.

Taylor, S. J . (y R. Bogdan)

2000 Introducción a los métodos cualitativos de investigación. La búsqueda de significados. Barcelona, Paidós.

Velasco, H. (y Á. Díaz de Rada)

2006 La lógica de la investigación etnográfica. Un modelo de trabajo para etnógrafos de escuela.

Madrid, Trotta.

Walker, $\mathrm{R}$.

1983 "La realización de estudios de casos en educación. Ética, teoría y procedimientos", en W. B.

Dockrell y D. Hamilton, Nuevas reflexiones sobre la investigación educativa (42-82). Madrid, Narcea.

Yacuzzi, E.

2005 "El estudio de caso como metodología de investigación: teoría, mecanismos causales, validación", Inomics, 1: 296-306.

Yin, R.

1989 Case Study Research. Design and Methods. London, SAGE.

Recibido: 19 abril 2012 | Aceptado: 20 junio 2012 | Publicado: 2012-06 